\title{
PARSEC SCALE PROPERTIES OF LOW POWER
}

\section{RADIO GALAXIES}

\author{
G. GIOVANNINI ${ }^{1,2}$, W.D. COTTON ${ }^{3}$, L. LARA $^{1,4}$, T. VENTURI $^{1}$ \\ 1 Istituto di Radioastronomia, CNR, Bologna, Italy \\ 2 Dipartimento di Astronomia, Universita' di Bologna, Italy \\ 3 NRAO, Charlottesville, VA, USA \\ ${ }^{4}$ Instituto de Astrofisica de Andalucia, CSIC, Granada, Spain
}

\section{Radio Morphology and Jet Properties}

We shortly discuss here the parsec scale information presently available on the extended low power radio galaxy (FR I, Fanaroff and Riley, 1974) NGC6251 (Jones and Wehrle, 1994) and on 11 radio galaxies from the sample presented in Giovannini et al. (1990).

We have used the observational data to derive constraints on the jet velocity and orientation with respect to the line of sight and will use these values to test the predictions of the unified scheme models (see also Venturi et al., this volume).

With the exception of 3C338, (a two-sided symmetric parsec scale jet), and of $1144+35$ (a complex structure), we have always an asymmetric parsec scale morphology (core emission and a one-sided jet) where the nuclear emission is always the dominant component. The one-sided parsec scale jets do not show large bends and are always oriented as the main kpc scale jet. This result, coupled with the constraints derived from the jet/counter jet brightness ratio (see also Venturi et al., this volume and Wrobel et al., this volume) and the radio core prominence (Giovannini et al. 1994 and 1995) is consistent with the expectations of unified models. In fact, in the FR I radio galaxies discussed here, observational data are in agreement with the presence of a parsec scale jet with an intrinsic Lorentz factor $\gamma \gtrsim 2$ viewed at angles larger than $30^{\circ}$, even if in a few cases we cannot exclude smaller 
angles with a lower jet velocity. This is consistent with FR I galaxies being the parent population of BL-Lac objects.

\section{Proper Motion}

The high jet velocity derived in the previous section, suggests that proper motion should be detectable in parsec scale jet of FR I radio galaxies, if these sources are monitored with a time gap of 1 - 2 years. However a few FR I galaxies have presently at least two observations at different epochs useful to look for the existence of a proper motion while a proper motion is firmly established in strong radio galaxies, quasars and BL-Lac objects (Vermeulen, this volume; Zensus, this volume).

The galaxy 3C274 (M87) shows evidence of stationary knots as well as structures moving at sub-relativistic velocities. Moreover, some substructures seem to have a superluminal speed (Biretta et al., 1995). NGC6251 could have both a stationary knot and one moving at $\mathrm{v} \sim 1.2 \mathrm{c}$ (Jones and Wehrle, 1994). The B2 galaxy $1144+35$ has a complex parsec scale structure with two unresolved flat spectrum regions which are moving one with respect to the other with an apparent velocity of $1.2 \mathrm{c}$ (Giovannini et al., 1995). The symmetric source 3 C338 shows a clear change in its radio structure and present data suggest a possible motion corresponding to an apparent velocity of 0.5c (Giovannini et al., 1995).

To better investigate this point, we obtained new maps of $3 \mathrm{FR}$ I radio galaxies: NGC315, 4C29.30 and 3C465. The comparison between the two different epoch maps shows that no proper motion is visible in any of these 3 sources. The derived velocity upper limits are $0.1 c, 0.7 \mathrm{c}$ and $0.3 \mathrm{c}$ for NGC315, 4C29.30 and 3C465, respectively. In this respect the lack of visible motion in FR I parsec scale jets, which are expected to move at a relativistic velocity, could reflect the presence of oblique shocks and/or more complex situations where the apparent knot velocity could be much lower than the jet velocity. This result could suggest that despite the relativistic velocity present in low and high power parsec scale jets, an intrinsic difference in the jet dynamic, could be present.

A Hubble constant $\mathrm{H}_{0}=100 \mathrm{~km} \mathrm{~s}^{-1} \mathrm{Mpc}^{-1}$ was used in this work.

\section{References}

Biretta, J.A., Junor, W. (1995) Proc. Natl. Acad. Sci. USA, 92, in press

Fanaroff, B.L., Riley, J.M. (1974) Month. Not. R. Astr. Soc., 250, 198

Giovannini, G., Feretti, L., Comoretto G. (1990) ApJ 358, 159

Giovannini, G., Feretti, L., Venturi, T., Lara L., et al. (1994) ApJ 485, 116

Giovannini, G., Cotton, W.D., Feretti, L. et al. (1995) Proc. Natl. Acad. Sci. 82, in press Jones, D.L., Wehrle, A.E. (1994) ApJ 427, 221 